\title{
WATCHING HORIZONTAL LIGHTNING PROPAGATION
}

\author{
By DENNIS G. BAKER* and ANITA BAKER-BLOCKER† \\ *Dept. of Atmospheric \& Oceanic Science, University of Michigan \\ †Advanced Science Consultants, Ann Arbor, USA
}

$\mathrm{T}$

HE speed of lightning is proverbial, extolled in such popular idioms as 'quick as a flash' and 'fast as greased lightning'. Yet the idea that some lightning propagates so slowly as to be visually perceived to move is not new. In 1887 , D'Abbadie described seeing the direction of propagation for a horizontal lightning flash that he estimated to be $6.7 \mathrm{~km}$ in length. One of his contemporaries debated D'Abbadie's claim, writing: $\because$. . this seeming recognition of direction must be an illusion of judgement based.upon our interpretation of the phenomena presented to the sight' (LeConte, 1887). More recently, trained meteorologists have observed slow moving horizontal cloud-to-cloud lightning. Bartlett (1970) stated: 'Even with the unaided eye, one is in no doubt about the direction in which any particular intracloud stroke had been propagated'. Krider (1974) described a very large air lightning discharge that he saw visually propagating an estimated $16 \mathrm{~km}$ in $1.0 \pm 0.5$ seconds, implying a velocity of $1-4 \times 10^{4}$ metres per second. Such a velocity, coupled with its large areal extent, makes horizontal lightning discharges among the fastest of all natural phenomena in which motion can be discerned by the unaided human eye. Yet the perception of motion of the phenomena involves more than just the ability of the eye to see the event; it involves the entire visual processing system, both eye and brain, in the recognition of what has occurred in the event.

During nocturnal thunderstorms, we have managed to observe and photograph lightning flashes with large horizontal components that we have observed propagating (Fig. 1). Our observations are in agreement with reports of a few other observers, as typified by McCutcheon (personal communication): 'I do not register that each channel lights up progressively from one end, only that there is a delay between the discharge of one group of parallel channels and the group beyond the interruption'. Unfortunately, our visual observations can be neither supported nor contradicted by our photographs of flashes in which we have observed propagation, probably, because these photographs represent time exposures. For this reason, we have investigated how the human visual processing system recognizes propagation when an observer witnesses long duration horizontal lightning.

\section{TEMPORAL AND SPATIAL FEATURES OF HORIZONTAL LIGHTNING}

Many good photographs of large horizontal lightning discharges have been taken, usually at night; the photograph of the widely branching cloud-to-air discharge in Fig. 1 is fairly typical. While still photographs can give some indication of spatial extent, and indicate the type of discharge, they are time exposures of an event with a very fast time frame. This limitation is somewhat overcome by use of videotape records. Such a record was examined by Brantley, et al. (1975), who found that horizontal extensions on the order of 100 metres occurred in about 10 per cent of the frames. The discharges in this videotape lasted an average of 310 milliseconds, and ranged in length from 1.2 to $3.7 \mathrm{~km}$, and had a mean propagation pause time of around 60 milliseconds with about 4 pauses per horizontal channel.

Long duration horizontal lightning flashes are almost certainly made up of multiple strokes. Malan (1956) found that 50 per cent of all flashes with durations ranging between 200 and 400 milliseconds were composed of 5 or more strokes. While the strokes may occur at intervals of 100 milliseconds or longer, the illumination of the lightning channel decays between strokes. Exceptionally long interstroke intervals favour dart-stepped leaders preceeding the later stroke (Malan, 1956). 


\section{FACTORS GOVERNING THE VISUAL OBSERVATION OF LIGHTNING}

One key difference between watching the flight of birds or airplanes and observing lightning flashes is the time period available for the observation. When watching events lasting in the range of seconds to minutes, an observer is able to judge motion on the relative motion of the object, coupled with a perception of eye and head movements (Brown, 1958). When the observer's eyes attempt to follow a moving target, the eyes move in a manner known as 'smooth pursuit' which is triggered after an initial stimulus period of about 125 milliseconds. Eye velocity can rarely match target velocity after smooth pursuit is evoked (Alpern, 1972), so the visual processing system triggers an eye movement called a 'saccade' which is a shift in gaze between two points equally distant from an observer. The reaction time for a saccade is about $\mathbf{2 0 0}$ milliseconds. This time frame works quite well when observing birds and airplanes, but fails when observing an event that might last on the order of 200-400 milliseconds; an important consideration since only half of all lightning flashes with five or more strokes last longer than 350 milliseconds (Malan, 1956).

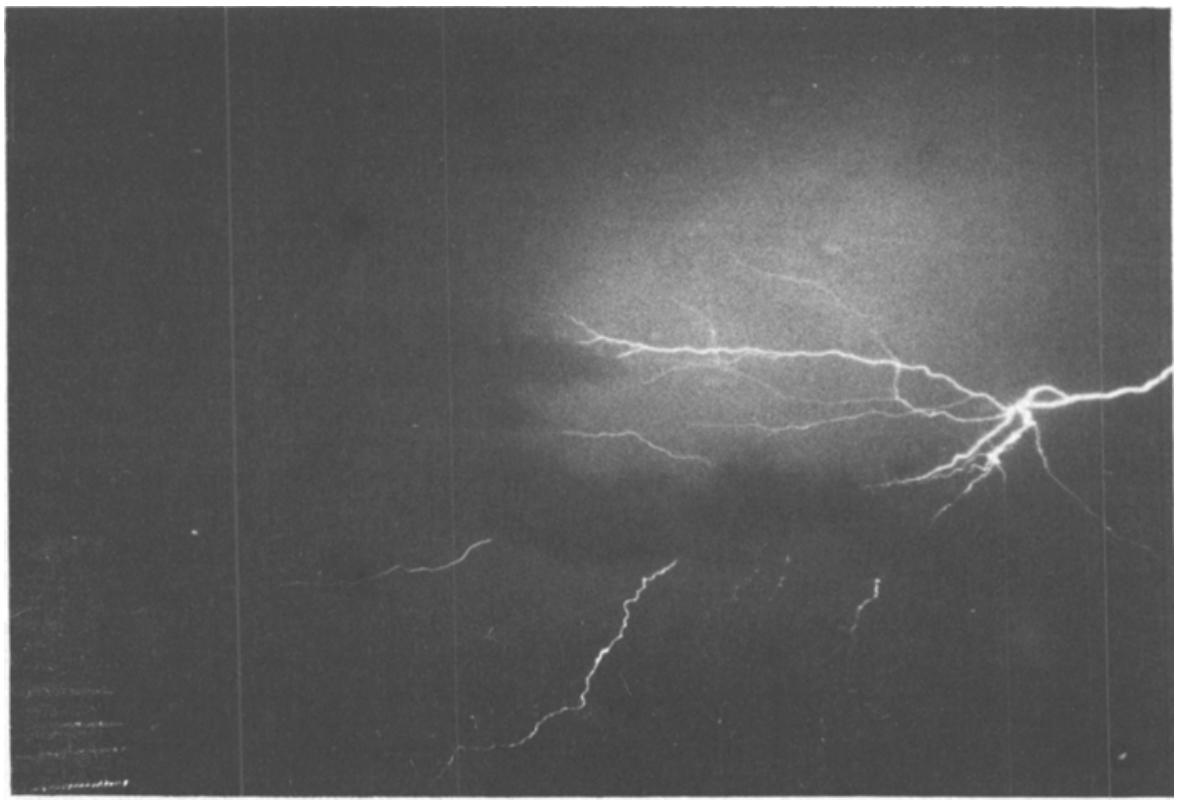

photograph by D. G. Baker and A. Baker-Blocker

Fig. 1 Cloud-to-air lightning discharge emanating from a cloud base of around $2 \mathrm{~km}$ height at 4.30 AM Eastern Daylight Time on 9 June 1985 over Ann Arbor, Michigan. At the time of the flash, a cold front had passed through and precipitation had ended at the ground

Other factors, largely related to the distance between the observer and the long duration lightning flash, are also important. For example, a horizontal lightning flash passing directly overhead at a height of 1800 metres and having a velocity of $2 \times 10^{4}$ metres per second would have an angular velocity of $585^{\circ}$ per second. The peak velocity of eye movement shifting between two positions is on the order of $830^{\circ}$ per second (Alpern, 1972), but such a shift is evoked only after a delay in the initial stimulus. The upper threshold for the detection of motion of a bright object against a dark background is around $36^{\circ}$ of visual angle per second (Brown, 1958). Thus it is most likely that an 
observer would be able to detect slow propagation only in large horizontal lightning flashes that were distant from the observer. Krider's (1974) flash would have to be at least $7 \mathrm{~km}$ distant for the observer to see the flash move through $<36^{\circ}$ of visual angle per second.

If an observer is fortunate enough to witness a long duration lightning flash with his eyes steadily fixating (with no head or eye movement), a sufficient condition for the visual perception of target movement and displacement only requires a shift in retinal focus (Matin, 1972). However, is what the observer sees really movement? What we perceived as propagation was succinctly put as '. . . a delay between the discharge of one group of parallel channels and the group beyond the interruption' by McCutcheon (personal communication). This observation supports a perception of temporal ordering, rather than the detection of the direction of movement.

Temporal ordering can be determined perfectly if there is a $\mathbf{3 0}$ millisecond pause between the onset of two targets (McKee and Taylor, 1984). Since Brantley, et al. (1975) found an average propagation pause time of 60 milliseconds, the time frame for the propagation of a horizontal lightning flash is more than adequate for the visual processing system of an observer to resolve temporal ordering in the sequence of the flash.

\section{CONCLUSIONS}

The visual observation of lightning propagation is possible in situations where the observer is able to watch long duration horizontal lightning flashes at a substantial distance. In such cases, what is perceived as propagation is a combination of two separate aspects of the phenomena: the extension of the flash perceived by a slight shift in retinal focus of the eyes, or possibly by some tracking movement of the eyes, and the temporal ordering of the flash, as the visual processing system recognizes the time delay in the illumination of the extending lightning channel. Temporal ordering of the channel illumination is not dependent on the observer being at a substantial distance from the flash, and is probably observed more frequently than is actual motion in the extension of the lightning flash. Visual observation of the propagation of horizontal lightning is at best serendipitous; the necessity for the observer to be watching the right area of the sky at the time when long duration horizontal lightning is occurring is a result not only of persistence but also luck.

\section{REFERENCES}

Alpern, M. (1972) Eye Movements. Handbook of Sensory Perception, 7, New York (Springer Verlag)

Bartlett, J. T. (1970) Intracloud Lightning. Weather, 25(7), pp. 316-318

Brantley, R. D., Tiller, J. A., and Uman, M. A. (1975) Lightning Properties in Florida Thunderstorms from Video Tape Records. J. Geophys. Res., 80, pp. 3402-3406

Brown, R. H. (1958) Influence of Stimulus upon the Upper Speed Threshold for the Visual Discrimination of Movement. J. Opt. Soc. Amer., 48, pp. 125-128

D'Abbadie, A. (1887) Lightning Flashes. Nature, 35, p. 342

Krider, E. P. (1974) An Unusual Photograph of an Air Lightning Discharge. Weather, 29(1), pp. 24-27

LeConte, J. (1887) Lightning Flashes. Nature, 35, p. 342

Malan, D. J. (1956) The Relation Between the Number of Strokes, Stroke Intervals and the Total Durations of Lightning Discharges. Pure \& Appl. Geophys., 34, pp. 224-230

Matin, L. (1972) Eye Movements and Perceived Visual Direction. Handbook of Sensory Perception, 7, New York (Springer Verlag)

McKee, S. and Taylor, D. G. (1984) Discrimination of Time: Comparison of Foveal and Peripheral Sensitivity. J. Opt. Soc. Amer., A/1, pp. 620-627 


\section{CUMULUS}

from a colour transparency by Marion Biggs

From Wier Wood Reservoir, near East Grinstead, West Sussex, looking east along a 'street' of cumulus clouds forming in a very strong westerly wind behind an intense depression. I230 BST, 24 March 1986

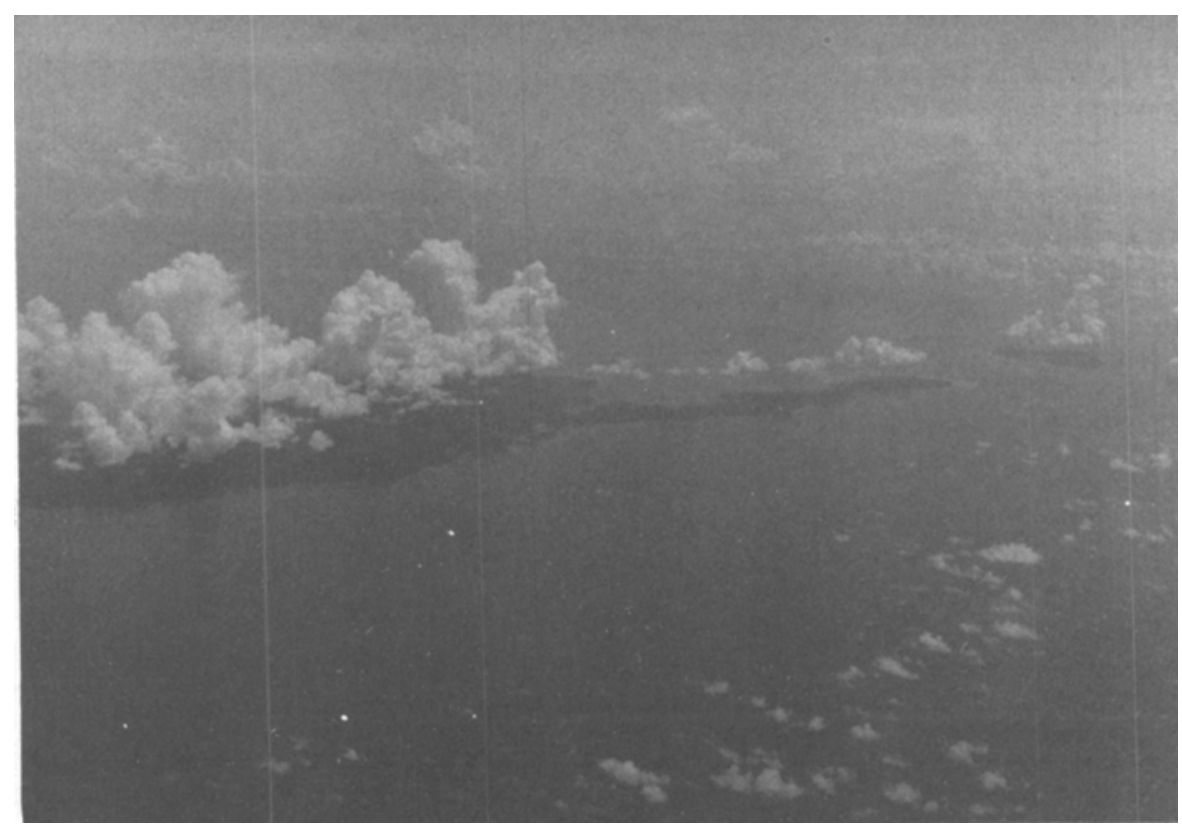

from a colour print by Keith Eaton Cloud formation over the Solomon Islands, from 35,000fi at 1100LT, 22 February 1984. San Cristobel is on the extreme right, Santa Ana on the extreme left 